\title{
Mapping the Spatial Variability of Sexually Transmitted Infections across Fiji Health Regions
}

\author{
Tayyab Shah ${ }^{1}$, Michaela Sidloski², and Elisa Toroca ${ }^{3}$ \\ ${ }^{1}$ University of Saskatchewan Canadian Hub for Applied and Social Research, 9 \\ Campus Drive, Saskatoon, SK, Canada \\ Email address: tayyab.shah@usask.ca
}

${ }^{2}$ Corresponding author: University of Saskatchewan School of Environment and

Sustainability, 117 Science Place, Saskatoon, SK, Canada

Email address: michaela.sidloski@usask.ca

Phone: 1 (306) 250-3213

${ }^{3}$ University of the South Pacific School of Geography, Laucala Bay Road, Suva, Fiji

Email Address: elisatoroca1098@gmail.com

\section{Abstract}

Introduction: Sexually transmitted infections (STIs) are a significant public health problem in countries within the South Pacific, including Fiji. If untreated, curable STIs such as chlamydia, gonorrhea, and syphilis can cause infertility, adverse outcomes in pregnancy, and can increase the risk of contracting HIV in infected individuals.

Methods: This research used cartographic software to map and analyze the spatial distribution of selected STIs across health regions in Fiji. Total rates of STIs, as well as the prevalence of gonorrhea and syphilis specifically, were examined for the years of 2007 and 2016 to determine how spatial distribution patterns have changed over this period, and how resources might currently be most effectively mobilized to address this public health issue.

Results/Discussion: Our findings suggest that while some specific regions with high prevalence rates for 2007 and 2016 should be targeted for intervention in the short term, lack of data collecting and reporting raises concerns about the accuracy of rate estimations in non-urban areas.

Conclusion: Analyzing the spatial distribution of the prevalence of STIs in a given population can better inform the development and implementation of intervention strategies at local scales, thus improving health outcomes for countries and their communities. Overall, consistent and transparent STI data collection and reporting procedures are necessary for effective long-term management and minimization of STI spread in Fiji. 
Keywords: STI prevalence, Fiji, spatial distribution, mapping

Michaela Sidloski and Tayyab I. Shah are geospatial researchers affiliated respectively with the School of Environment and Sustainability and the Canadian Hub for Applied and Social Research at the University of Saskatchewan, Canada. Elisa Toroca is affiliated with the University of the South Pacific, Fiji. For correspondence, contact Michaela Sidloski by email at michaela.sidloski@usask.ca. 


\section{Introduction}

Sexually Transmitted Infections (STIs) are a global health concern that create social and economic challenges for governments and their populations. Left untreated, common curable STIs such as chlamydia, gonorrhea, and syphilis increase the risk of many co-morbidities including infertility, complications in pregnancy and childbirth, and human immunodeficiency virus (HIV) (Cliffe et al., 2008). In Pacific countries, factors that typically contribute to the spread of these diseases include low rates of condom use during sexual activity, having multiple sexual partners, practicing commercial sex, and having limited knowledge about STIs (Fiji Ministry of Health, 2013a; Cliffe et al., 2008). High prevalence of STIs in populations typically considered low-risk have raised concern about rates of undiagnosed and untreated STIs in Pacific countries in the past decade (Cliffe et al., 2008). In more recent years, disproportionate rates of STI in youth and particularly indigenous youth populations have raised concern in Pacific countries, including Fiji (Mitchell, 2015).

The Fiji Ministry of Health (2013a, p. 24) states that "[c]hlamydia rates in both Fiji and Vanuatu are amongst the highest in the world." At 26.8\% in 2009, Fiji had the highest chlamydia prevalence rate for pregnant women when compared to seven other countries, including Papua New Guinea, India, and Thailand (Fiji Ministry of Health, 2013b). However, access to information about STIs in Fiji is limited. Few opportunities for sexual health education result in low public awareness of STIs like chlamydia, even among health staff (Fiji Ministry of Health, 2013a). Some have attributed this lack of awareness to the centralized nature of health services in the country, which results in a very small subset of health care workers dealing with chlamydia and related conditions on a regular basis (Fiji Ministry of Health, 2013a).

In Fiji, the prevalence of syphilis (2.7\%) and gonorrhea (2.2\%) have also greatly exceeded the $1.5 \%$ average rate for the Western Pacific Region (Fiji Ministry of Health, 2013a). Although these do not constitute epidemic rates, the impacts have already been felt by Fiji's health and economic sectors. For example, due to the prevalence of STIs and increasing rates of HIV infection in neighbouring countries, the Fiji Ministry of Health has identified that the country is "at risk of having a possible rapid increase of HIV resulting in an epidemic" (Fiji Ministry of Health, 2013b, p. 36). With respect to economic impacts, the annual cost of implementing the Republic of Fiji's National Strategic Plan on HIV and STIs nearly doubled from 2012 to 2015. In 2012, FJD 4.7 million was invested in the programme. The cost increased to FJD 8.9 million in 2015 when adjusted for inflation (Fiji Ministry of Health, 2016). Between 2012 and 2015, preventing HIV \& STI transmission alone had an estimated cost of FJD 15.0 million, accounting for $56 \%$ of the plan's total implementation cost of FJD 26.6 million over the same time period (Fiji Ministry of Health, 2016). The increasing costs associated with STI prevention and intervention in Fiji speaks to the importance of developing effective solutions around managing the spread of STIs in this region. 
Geographic Information Systems (GIS) is an emerging tool for public health intervention that can consolidate large quantities of data from diverse sources into convenient and understandable formats. Spatial data visualization can be immensely valuable when used to inform decision-makers, public health workers, and society at large about health-related issues (Geanuracos et al., 2007). In a review of GIS use in the public health sector, Nykiforuk and Flaman (2011) conclude that GIS is a highly valuable tool with myriad potential applications in the public health sphere. Appropriately, geospatial mapping approaches are increasingly used to examine STI distribution patterns at different geographic scales (Law et al. 2004; Kerani et al. 2005; Cuadros et al. 2017; Lai et al. 2019). For instance, (Law et al. 2004) analyzed the spatial variability of four STIs in Wake County, North Carolina, USA in order to optimize the distribution of resources for intervention and prevention.

The spatial distribution of STIs has not yet been analyzed in the Fiji Islands despite the fact that STI cases are monitored and reported regularly by most health facilities. As knowledge about the social and economic impacts of STIs in these regions becomes increasingly available, the value of investigating the spatial distribution of STIs becomes clear: GIS analysis can inform the development of more effective treatment and prevention strategies at local scales. Therefore, this research examines the spatial distribution of the prevalence rate of STI cases (number of cases per 1000 people) in Fiji reported by the Ministry of Health. Specifically, the objectives of this research are: (1) to map the spatial distribution of the rates of gonorrhea and syphilis across Fiji health regions, and (2) to identify how the spatial distribution varies over ten years by comparing rates of infection between 2007 and 2016.

\section{Methods}

Fiji is a lower-middle-income country, with a population of 884,887 in 2017 (Fiji Bureau of Statistics, 2018). The majority of the population are of reproductive age, while $20 \%$ of all Fijians are between 15 and 24 years of age (Fiji Ministry of Health, n.d.). Cases of STIs reported from 2007 to 2016 were acquired from the Fiji Ministry of Health in spreadsheet format. A separate file for each year was provided, including the following variables: STI cases at the health facility level disaggregated by STI type, health facility name, and the province of facility location. Spatial data about health region boundaries that represent health services' regions were collected from the University of the South Pacific GIS Lab. Fiji databases were used to map the variables of interest. This health region layer was prepared using the 2007 enumeration area (EA-the smallest census unit of analysis) boundaries retrieved from the Fijian Bureau of Statistics. Population data from the 2017 census was not available at this level of analysis.

Prevalence is a measure often used to represent the extent to which a population of interest is affected by a given disease; Noordzij et al. (2010, 
p. c18) term this "the burden of a disease." It is commonly used in disease mapping (Cuadros et al. 2017; Géopogui et al. 2018; Angarita-Fonseca et al. 2019) primarily due to its ability to illuminate target areas that could benefit from prioritization in resource distribution (Noordzij et al., 2010). In order to calculate the prevalence rate of STIs in Fiji health regions, the authors first combined separate files containing STI data by year into a single document. By consulting various data sources, including Google Maps and the Fiji Ministry of Health website, the geographic locations (a set of geographic coordinates) of the health facilities were identified based on their addresses. The researchers were unable to match some of the facilities to a discrete location (5 out of 138 , or $3.6 \%$ ) due to these facilities providing an incomplete or invalid address, or because the valid address that they provided was not available through Google Maps. Next, using the overlay analysis tool, 133 health facilities were spatially joined with the health services layer to link the information to its respective health region. To prepare data at the health region level, the attribute table of the health facilities layer was exported into Microsoft Excel. Using the PivotTable tool available in Microsoft Excel, STI information was aggregated to the health region level. Using a unique identification code, the aggregated STI data was linked to a file containing geospatial information about the location of each health region.

Using the 2007 population data, STI prevalence for each health region was calculated for the years 2007 and 2016. Specifically, the prevalence of gonorrhea and syphilis, as well as the total STI prevalence (including gonorrhea, chlamydia, syphilis, and congenital syphilis) was calculated for each health region. Using ArcMap 10.6 software, maps were produced displaying STI prevalence in each health region. The prevalence rate represents the number of STI cases per 1000 population within each health services area. For this research, thematic maps are used to depict the prevalence rate. A thematic map is a type of map that portrays the spatial distribution pattern of a variable of interest across an area of interest. To maintain comparability across maps, prevalence values were manually classified into the following four categories for all maps: 0.00 (no cases per 1000 people), 0.01 to $2.50,2.51$ to 5.00 , and more than 5.00 cases per 1000 people. The health facilities in some health regions were non-reporting facilities. These regions were symbolized in grey to distinguish them from those health regions that did have reporting health facilities but did not report any STI cases.

\section{Results and Discussion}

Figure 1 shows that total STI cases reported within Fiji's four major administrative divisions have decreased somewhat since 2007. The distribution of cases within each administrative division remains relatively constant, with the northern and western administrative regions appearing to fluctuate the most in total STI cases over these years. Figure 2 illustrates the distribution of STIs by age cohort in 2007 and 2016. The figure shows the combined total number of cases from 2007 and 2016, revealing that youths between 20 and 29 years old are the most likely to 
suffer from gonorrhea and syphilis by a large margin. Fijian infants below the age of 1 year are also strikingly susceptible to congenital syphilis. It is assumed that the unknown age category is comprised of patients who were not willing to reveal their age during a check-up or whose age was not recorded by the health practitioner. Table 1 provides a more detailed examination of the STI rates by including the prevalence rate of each type of STI to allow for better comparability across regions. While rates of the STIs included in this study have consistently declined in the central and western regions of the country between 2007 and 2016, they have consistently increased in the eastern and southern regions during the same period. This trend can be seen throughout the table, except in the case of syphilis, which shows a decrease in the western and central areas but no notable change in the eastern and southern regions between 2007 and 2016. The western and central regions have significantly larger populations than their eastern and southern counterparts, suggesting that intervention strategies in the past decade seem to have had more success in the more heavily populated areas of the country. This may be due to the fact that more dispersed populations are generally less likely to benefit from intervention and prevention programmes. For example, Mitchell (2015, p. 17) has called for improved "range and reach of current [sexual and reproductive health] services" in Fiji, as the availability of these services is limited in areas outside of major centres.

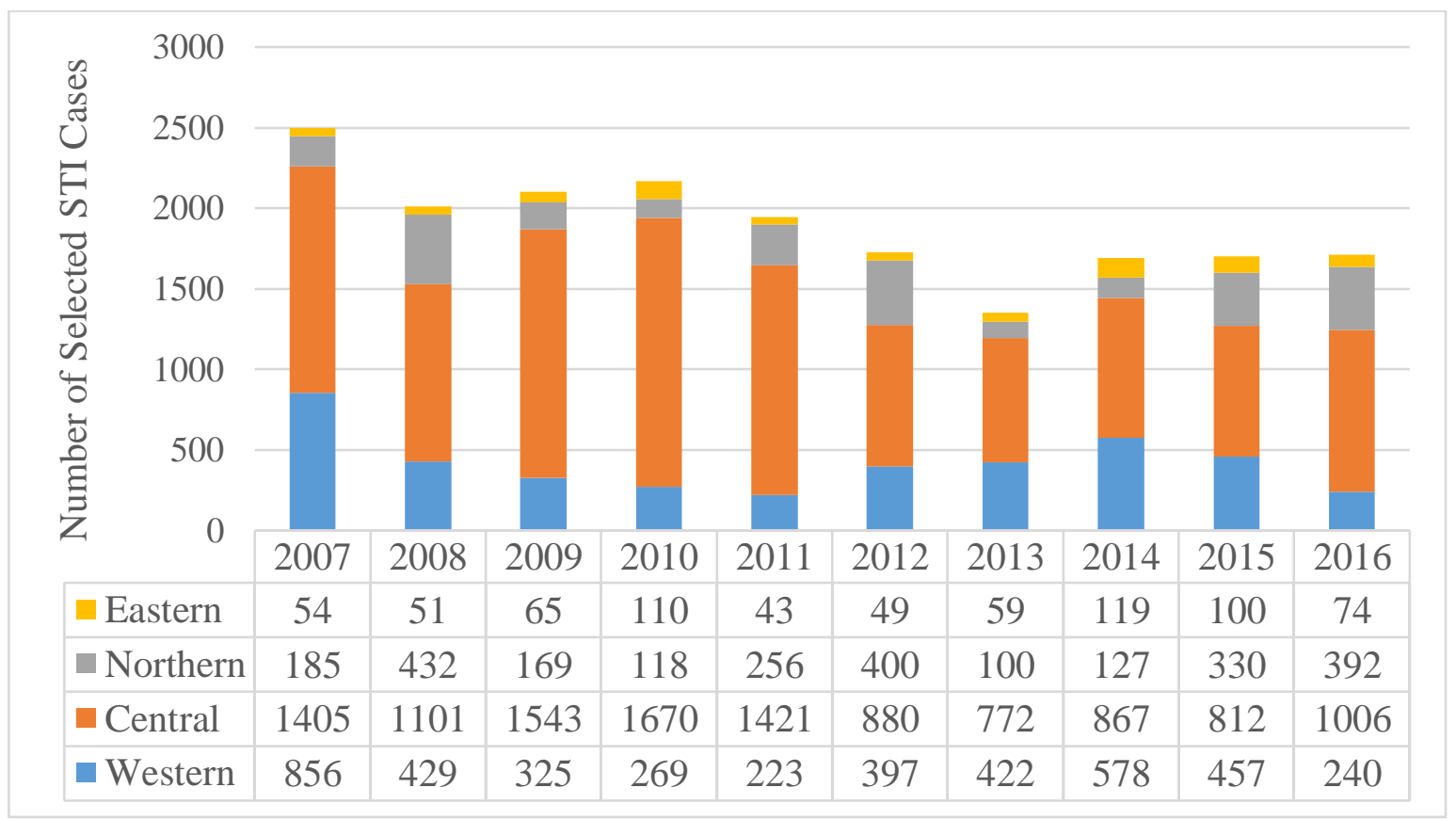

Figure 1. The number of STI cases (including gonorrhea, chlamydia, syphilis, and congenital syphilis) from 2007 to 2016 across Fiji's four major administrative divisions. (Source: Fiji National Notifiable Disease Surveillance System, 2019) 


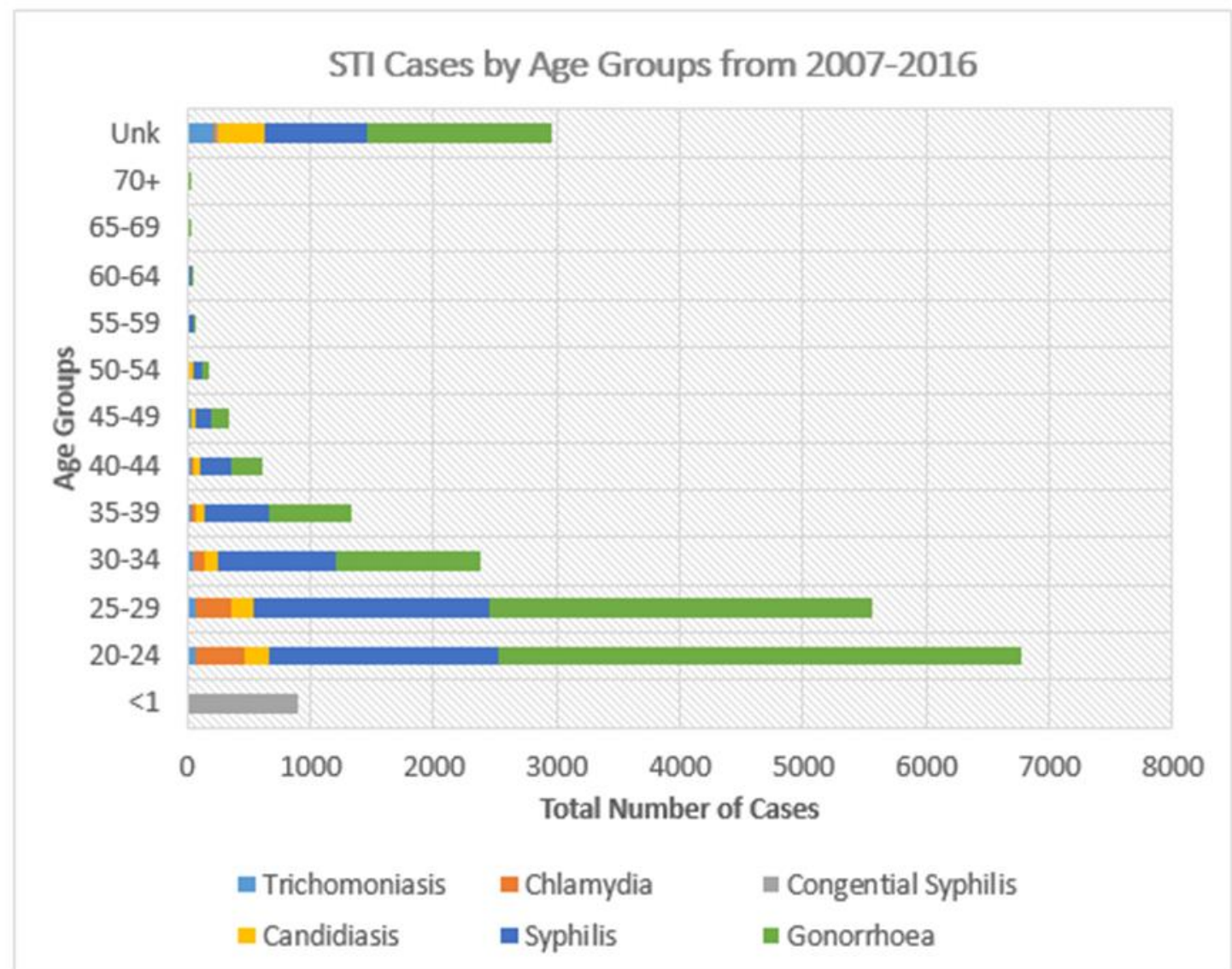

Figure 2. Aggregated 2007 and 2016 STI cases by STI type in Fiji. Unk: unknown ages - missing age information

(Source: Fiji National Notifiable Disease Surveillance System, 2019)

Table 1 presents a summary of STI cases and prevalence (per 1000 population) in 2007 and 2016 within Fiji's four major administrative divisions. The grey shaded cells represent the cases and prevalence of all selected STIs, while the unshaded cells display values for syphilis and gonorrhea specifically. As in the maps discussed below, it is notable that population data for 2016 was unavailable at the enumeration area level, so 2007 population data was used for both years. This means that prevalence rates for 2016 are likely slightly overestimated, as the country's total population has increased by $5.7 \%$ since the 2007 census, and the authors were unable to account for this slight population increase in this research (Fiji Bureau of Statistics, 2018). However, as the vast majority of this growth took place in urban areas (Fiji Bureau of Statistics, 2018), the areas most likely to be affected by prevalence overestimation in these results are those containing densely populated urban settlements. 
Table 1. Summary of 2007 and 2016 STI cases and prevalence rates (per 1000 population) across four major administrative divisions in Fiji. (Source: Fiji National Notifiable Disease Surveillance System, 2019)

\begin{tabular}{|c|c|c|c|c|c|c|c|c|c|c|c|c|c|c|}
\hline \multicolumn{3}{|c|}{ 1 } & \multicolumn{2}{|c|}{$\begin{array}{l}2007 \\
\text { Selected } \\
\text { STIs }\end{array}$} & \multicolumn{2}{|c|}{$\begin{array}{l}2016 \\
\text { Selected } \\
\text { STIs }\end{array}$} & \multicolumn{2}{|c|}{$\begin{array}{c}2007 \\
\text { Syphilis }\end{array}$} & \multicolumn{2}{|c|}{$\begin{array}{c}2016 \\
\text { Syphilis }\end{array}$} & \multicolumn{2}{|c|}{$\begin{array}{c}2007 \\
\text { Gonorrhea }\end{array}$} & \multicolumn{2}{|c|}{$\begin{array}{c}2016 \\
\text { Gonorrhea }\end{array}$} \\
\hline 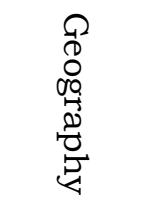 & 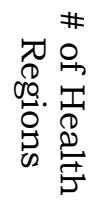 & 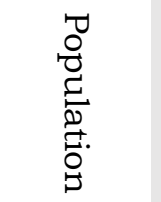 & 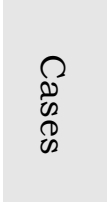 & 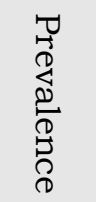 & 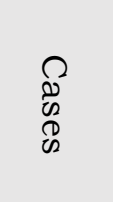 & 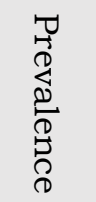 & 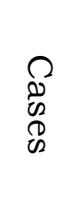 & 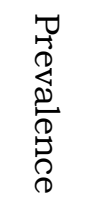 & 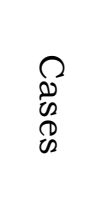 & 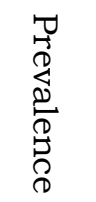 & 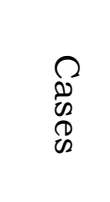 & 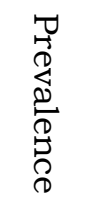 & 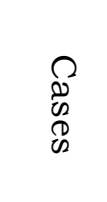 & 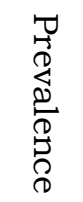 \\
\hline Western & 20 & 302,756 & 856 & 2.83 & 240 & 0.79 & 366 & 1.21 & 128 & 0.42 & 489 & 1.62 & 110 & 0.36 \\
\hline Central & 19 & 337,905 & 1,405 & 4.16 & 1,006 & 2.98 & 654 & 1.94 & 338 & 1.00 & 720 & 2.13 & 664 & 1.97 \\
\hline Northern & 16 & 125,641 & 185 & 1.47 & 392 & 3.12 & 73 & 0.58 & 46 & 0.37 & 112 & 0.89 & 333 & 2.65 \\
\hline Eastern & 13 & 32,823 & 54 & 1.65 & 74 & 2.25 & 13 & 0.40 & 13 & 0.40 & 41 & 1.25 & 61 & 1.86 \\
\hline
\end{tabular}

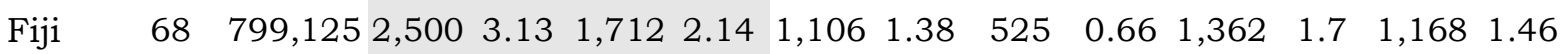

The spatial distribution of total STI prevalence (including gonorrhea, chlamydia, syphilis, and congenital syphilis), as well as gonorrhea and syphilis prevalence at health region levels, are shown in Figures 3-5 respectively. Each of the following figures contains two prevalence maps (for 2007 and 2016) along with an inset map showing the Greater Suva Area. The legend for each map delineates the range of prevalence rates within each colour category. The number of health regions falling within each category is also shown in parentheses. Grey areas represent health regions for which data was unavailable. 


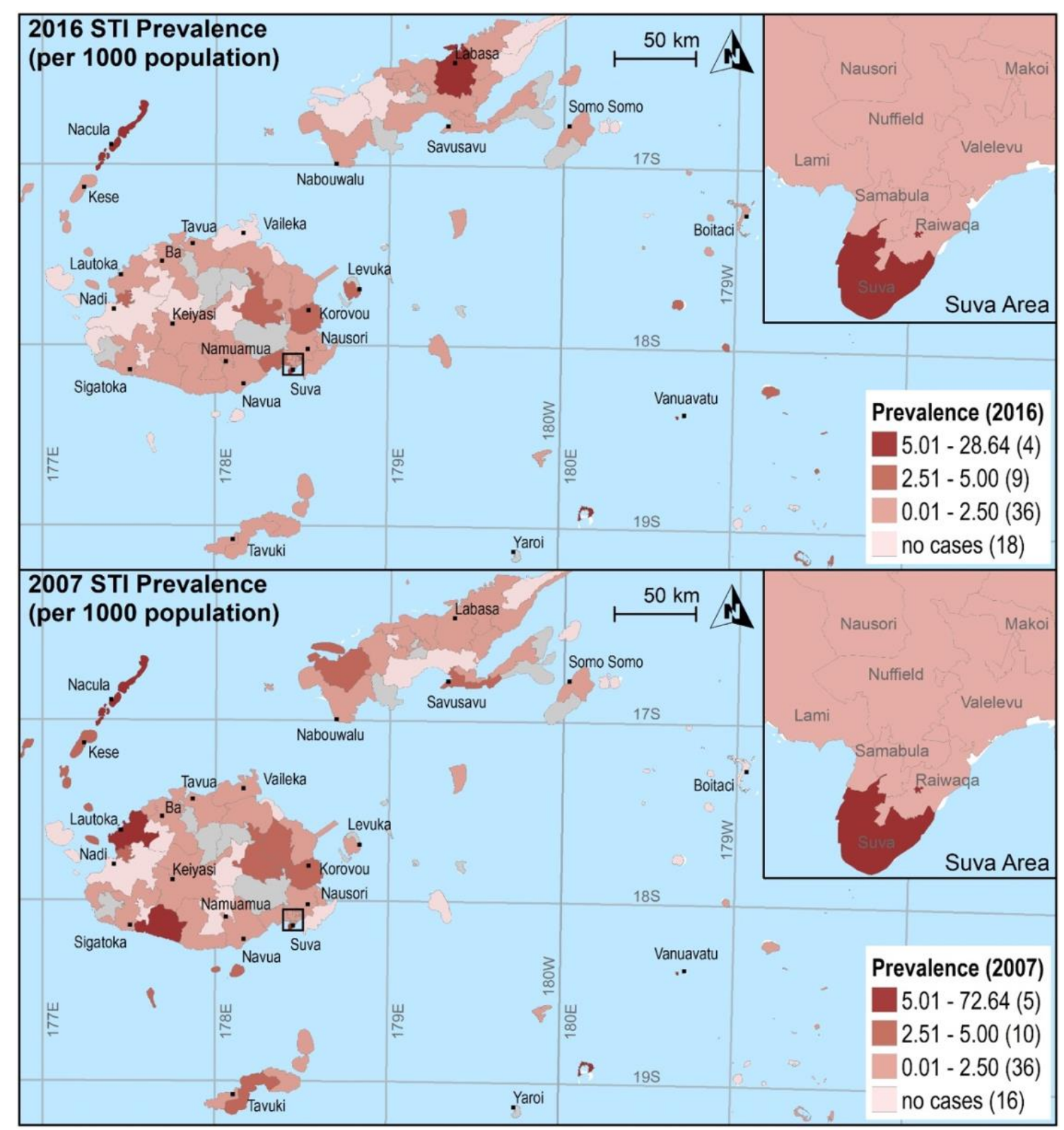

Figure 3. Selected STIs (gonorrhea, syphilis, chlamydia, and congenital syphilis) distribution across Fiji health service regions between 2007 and 2016. Grey areas represent health regions without reporting health facilities.

The maps in Figure 3 display the spatial distribution of all selected STIs in 2007 and 2016. In 2007, 15 health service regions reported high STI prevalence rates of greater than 2.50 per 1000 people. The majority of the most strongly affected areas were concentrated in the western and northern regions of the country. Meanwhile, 36 regions had relatively low prevalence rates, and 16 reported zero cases. By 2016, only two health service regions moved from the highest category to reporting less than 5.00 cases per 1000 people. Suva and the Yasawa Islands (Kese and Nacula) remained above this rate, and the Labasa area shifted into the highest category as well. However, the highest rate reported in the country decreased significantly from 72.64 in 2007 to 28.64 in 2016. Regions for which data was unavailable tend to be centrally located on the more populated islands, and tend to be near health service regions 
with relatively high prevalence rates; for example, those in the interior of Viti Levu (Navosa and Naitasiri) and Vanua Levu. The grey areas represent health regions without reporting health facilities. These health regions are located in the interiors of Viti Levu and Vanua Levu and have sparse populations. Collecting and reporting data on STI cases in these regions is critical to producing a more complete and robust understanding of the spatial trends in STI prevalence in Fiji.

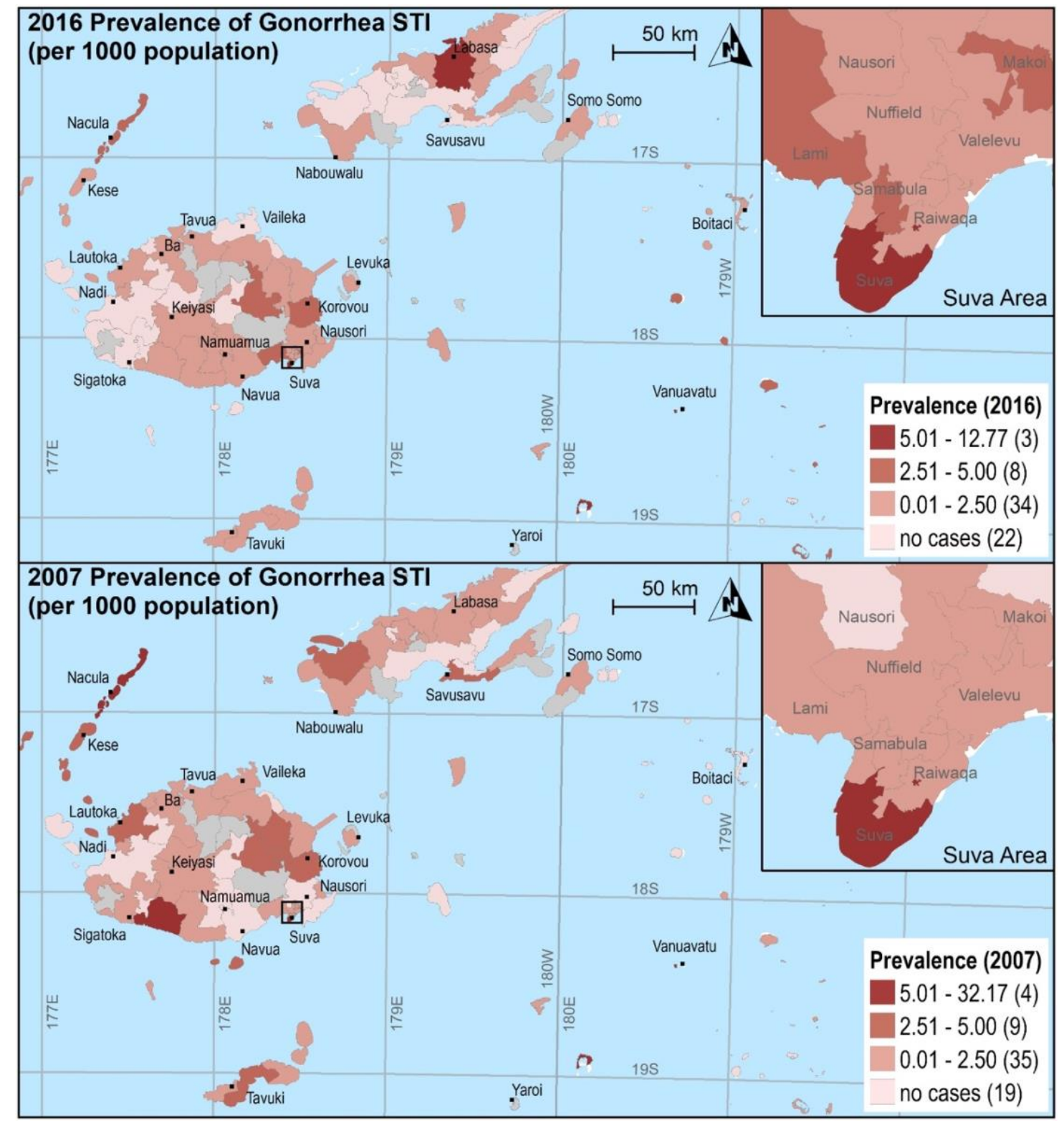

Figure 4. Gonorrhea prevalence rates across Fiji health service regions between 2007 and 2016.

Figure 4 shows that the distribution of gonorrhea prevalence rates shifted slightly between the years of 2007 and 2016 as well. Similar to the total STI case distribution, with the western region showing the most substantial decrease over time. Once again, the Suva area maintained a prevalence of greater than 5 cases per 1000 people during this time, and prevalence in the Labasa area increased to this category as well. The highest prevalence rate of all health regions decreased substantially from 
32.17 in 2007 to 12.77 in 2016 . The prevalence of syphilis, displayed in Figure 5, is significantly lower than that of gonorrhea throughout this period, with only one health region falling into the highest category in 2007 , and two in 2016. While rates of syphilis are generally lower across the country than rates of gonorrhea, the Suva area shows a particularly high prevalence rate in both years (38.44 in 2007 and 17.65 in 2016). Notably, Toyota Island's prevalence rate increased from zero cases in 2007 to the highest category in 2016. This could be due to an increase of testing capacity on this island, or due to a vast increase in infections during this time.

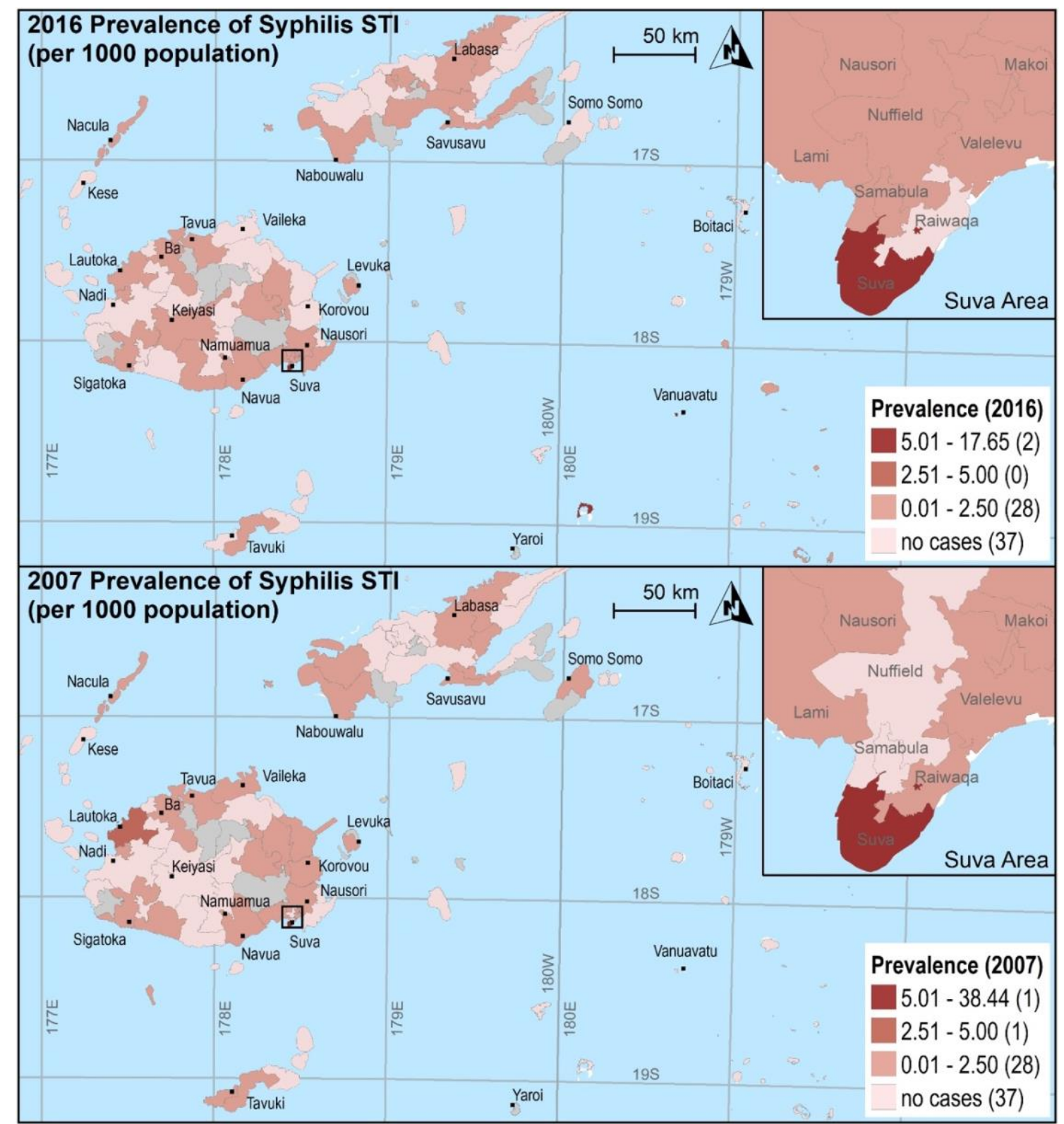

Figure 5. Syphilis prevalence rates across Fiji health service regions between 2007 and 2016.

One of the challenges encountered in this research was inconsistent data collection. Fiji's lack of procedural regulation in STI test administration 
and lack of a centralized reporting database (Mitchell, 2015) presented some challenges in interpreting these results. Further, health and demographic data on patients who had tested positive for STIs were unavailable for this research. This means that we were unable to determine on what basis STI tests were administered during the period under study. Therefore there is a possibility that sampling bias played a role in which populations generally accessed testing and treatment during this period. Further, STIs such as chlamydia have been recorded at unusually high rates in Pacific populations that are typically considered to be low-risk (i.e., pregnant women under the age of 25) (Cliffe et al., 2008). This suggests that STI rates may be underreported in areas for which testing is not highly accessible or performed routinely. Since many of the STIs studied herein may be present even in asymptomatic persons, we cannot ascertain what portion of the population may be infected but remain untested and untreated.

\section{Conclusion}

STIs have a high impact on the communities of Fiji. As this research demonstrates, these impacts do not impact all Fijians equally but affect specific populations and geographic areas disproportionately. In order to most effectively address this pressing issue, further research and several governmental actions are required. First, intervention programmes should be targeted to areas that have displayed increasing STI prevalence over the past decade (such as Labasa), as well as those that have maintained some of the highest prevalence rates consistently over this period (such as the Suva area and the Yasawa Islands). While it is clear that densely populated urban areas in Fiji tend to report higher STI rates, the lack of access to routine testing in remote and rural areas calls the accuracy of the relatively low prevalence in these areas into question. To optimize the accuracy of further research and STI reporting in Fiji, census data, and STI case reporting should follow consistent procedures across the country and across time. Overall, this research suggests that STIs in Fiji constitute a severe social, economic, and public health issue that should not go unaddressed. Developing targeted interventions based on the geographic distribution of prevalence rates across the country may be helpful in addressing this issue short-term. Still, universally accessible national interventions should be prioritized to manage this issue effectively into the future.

Future research directions could focus on spatial analyses of specific STIs with a significant presence in Fiji that were not examined here, such as chlamydia or HIV. Similar analyses conducted at broader scales (i.e., at the country level, including countries throughout the South Pacific) would also contribute to the current shortage of knowledge on the spatial patterns of STI prevalence in this part of the world. This research also illustrates the need for further investigation of where and how STI intervention programmes in Fiji are currently being implemented, and how access to these and other reproductive health services might be maximized. 


\section{Limitations}

This research was limited by the fact that population data at the EA level was available from the 2007 Fiji Census but not from the 2017 census at the time that this research was conducted. Due to the scarcity of data, a population estimate for 2016 was calculated at the health region level, but not for smaller areas. Therefore, the accuracy of the 2016 prevalence rates may have been compromised by changes in demographics that the researchers were unable to account for.

\section{Ethical Considerations}

Due to the sensitivity of health-related information, the data used in this research (provided by the Fiji Ministry of Health) was not linked to any personal patient information.

\section{Conflict of Interest}

The authors have no conflicts of interest to report.

\section{Acknowledgements}

The authors would like to acknowledge the generosity of the University of the South Pacific for providing access to research facilities and data. They would also like to express appreciation to the Fiji Ministry of Health for providing data that made this research possible. 


\section{References}

Angarita-Fonseca, A., Trask, C., Shah, T., \& Bath, B. (2019). Stable prevalence of chronic back disorders across gender, age, residence, and physical activity in Canadian adults from 2007 to 2014. BMC public health, 19(1), 1121. doi:10.1186/s12889-019-7395-8

Cliffe, S.J., Tabrizi, S., \& Sullivan, E.A. (2008). Chlamydia in the Pacific Region, the silent epidemic. Sexually Transmitted Diseases, 35(9), 801-806. doi:10.1097/OLQ.0b013e318175d885.

Cuadros, D. F., Li, J., Branscum, A. J., Akullian, A., Jia, P., Mziray, E. N., \& Tanser, F. (2017). Mapping the spatial variability of HIV infection in Sub-Saharan Africa: Effective information for localized HIV prevention and control. Scientific reports, 7(1), 1-11. doi:10.1038/s41598-017-09464-y.

Fiji Bureau of Statistics. (2018). 2017 Population and Housing Census: Release 1. Retrieved from: https:/ /www.statsfiji.gov.fj/index.php/ statistics / 2007-census-of-population-and-housing

Fiji Ministry of Health. (2013a). Assessment of the current STI response in Fiji and Vanuatu, and exploration of models to strengthen access to STI and HIV services by maximizing the value of client interactions with health services. Retrieved from:

http://old.aidsdatahub.org/assessment-current-sti-response-fijiand-vanuatu-and-exploration-models-strengthen-access-sti-and

Fiji Ministry of Health. (2013b). Global AIDS progress report 2013: Fiji islands. Retrieved from: https://www.unaids.org/sites/ default/files/country/documents/FJI_narrative_report_2014.pdf

Fiji Ministry of Health (2016). Republic of Fiji national strategic plan on HIV and STIs, 2012-2015. Retrieved from:

http:/ / www.health.gov.fj/wp-content/uploads/2014/05/6 _National-Strategic-Plan-on-HIV-and-STI_2012-2015.pdf

Fiji Ministry of Health. (n.d.). Republic of Fiji national strategic plan on HIV and STIs, 2016-2020. Retrieved from: https: / /www.aidsdatahub.org/sites/default/files/publication/ Republic_of_Fiji_National_Strategic_Action_Plan_on_HIV_and_STI s_2016-2020.pdf

Géopogui, A., Badila, C.F., Baldé, M.S., Nieba, C., Lamah, L., Reid, S.D., ... \& Amon, J.J. (2018). Baseline trachoma prevalence in Guinea: Results of national trachoma mapping in 31 health districts. PLOS Neglected Tropical Diseases, 12(6), e0006585. doi:https://doi.org/10.1371/journal.pntd.0006585

Kerani, RP, Handcock, M.S., Handsfield, H.H., \& Holmes, K.K. (2005). Comparative geographic concentrations of 4 sexually transmitted infections. American Journal of Public Health, 95(2): 324-330. doi:10.2105/ajph.2003.029413.

Lai, P. T., Wilson, J., Wu, H., Jones, J., \& Dixon, B. E. (2019). Measuring and visualizing chlamydia and gonorrhea inequality: An informatics approach using geographical information systems. Online Journal of Public Health Informatics, 11(2), e8. doi:10.5210/ojphi.v11i2.10155.

Law, DCG, Serre, M.L., Christakos, G., Leone, P.A., \& Miller, W.C. 
(2004). Spatial analysis and mapping of sexually transmitted diseases to optimise intervention and prevention strategies. Sexually Transmitted Infections, 80, 294-299. Doi: $10.1136 /$ sti.2003.006700

Mitchell, E. (2015). The Fiji national response to HIV and sexually transmitted infections: Mapping key successes and challenges. Journal of Pacific Studies, 35(1), 5-25. Retrieved from: https://www.researchgate.net/profile/Elke_Mitchell/publication/ 281397309_The_Fiji_national_response_to_HIV_and_sexually_tra nsmitted_infections_Mapping_key_successes_and_challenges/link s/59def81caca27247d7973ed5/The-Fiji-national-responseto-HIV-and-sexually-transmitted-infections-Mapping-keysuccesses-and-challenges.pdf

Nykiforuk, C.I.J., \& Flaman, L.M. (2011). Geographic information systems (GIS) for health promotion and public health: A review. Society for Public Health Education, 12(1), 63-73. doi:10.1177/1524839909334624 